\title{
A SÍNDROME DO CHOQUE CIRCULATÓRIO
}

\author{
CIRCULATORY SHOCK SYNDROME
}

Flávio Marson ${ }^{1}$; Gerson A. Pereira Jr. ${ }^{1}$; Antonio Pazin Filho ${ }^{1}$ \& Anibal Basile-Filho²

\begin{abstract}
${ }^{1}$ Médicos intensivistas, ${ }^{2}$ Docente e Chefe da Disciplina de Terapia Intensiva do Departamento de Cirurgia, Ortopedia e Traumatologia da Faculdade de Medicina de Ribeirão Preto da Universidade de São Paulo.

CorRespondÊnCIA: Prof.Dr. Anibal Basile-Filho - Disciplina de Terapia Intensiva, Departamento de Cirurgia, Ortopedia e Traumatologia da FMRPUSP - Hospital das Clínicas - $9^{\circ}$ Andar- Campus Universitário - CEP: 14048-900 - Ribeirão Preto - SP. Fone: (016) 633-0836 ou 602-2593 - Email: abasile@.fmrp.usp.br
\end{abstract}

MARSON F et al. A A síndrome do choque circulatório. Medicina, Ribeirão Preto, 31: 369-379, jul./set. 1998.

RESUMO: O choque circulatório é marcado por reduções críticas na perfusão tecidual, provocando alterações sistêmicas graves, com comprometimento da função celular e orgânica, com alto índice de mortalidade. O diagnóstico e a instituição de medidas terapêuticas devem ser precoces e baseados na resposta individual de cada paciente, dando-se ênfase ao suporte ventilatório e hemodinâmico. O conhecimento profundo da sua fisiopatologia norteia as decisões terapêuticas, uma vez que através dela se estabelecem objetivos a serem atingidos. Diversas condutas recentes estão sendo pesquisadas em pacientes com choque circulatório. Estas medidas incluem reposições volêmicas, simples, soluções substitutas de hemácias e terapias dirigidas aos mediadores (citoquinas, endotoxinas, prostaglandinas, leucotrienos e fator de ativação plaquetária).

UNITERMOS: Choque. Hemodinâmica.

\section{INTRODUÇÃO}

O diagnóstico e o tratamento do choque circulatório constituem-se num dos principais problemas nas unidades de terapia intensiva. Apesar dos avanços na compreensão da fisiopatologia do choque e de melhorias importantes na terapêutica, a mortalidade mantém-se elevada. Nos estágios iniciais, a ressuscitação vigorosa pode corrigir a hipoxia tecidual, permitindo a reversão do estado de choque. Entretanto, se a perfusão e o transporte de oxigênio continuarem diminuídos, a lesão celular pode tornar-se irreversível. Mesmo quando a terapia inicial parece ser adequada, o estado de hipermetabolismo e a síndrome da disfunção de múltiplos órgãos podem ocorrer. Deste modo, o sucesso terapêutico depende do diagnóstico precoce, ressuscitação rápida e terapia agressiva, dirigida à causa do estado de choque. O presente artigo de revisão discute a fisiopatologia do choque cir- culatório, seus aspectos clínicos e as bases do seu tratamento, com maior ênfase aos tipos de soluções utilizadas para a reposição volêmica.

\section{FISIOPATOLOGIA}

O choque circulatório é marcado por reduções críticas na perfusão tecidual. A hipoxia tecidual compromete a atividade metabólica, celular e, finalmente, as funções celulares e orgânicas. A resposta inicial do sistema cardiovascular às reduções graves na perfusão tecidual é um conjunto complexo de reflexos que servem para manter o tônus vascular e o desempenho cardíaco. Ocorre ativação do sistema simpático que eleva a frequiência e a contratilidade cardíacas. Há liberação de catecolaminas, vasopressina e angiotensina, que aumentam o tônus vascular e arteriolar, aumentando o volume sangüíneo central, o retorno venoso e a pressão sangüínea. Concomitantemente, o 
fluxo sangüíneo é direcionado para o cérebro e coração. À medida que o choque progride, os mecanismos compensatórios entram em falência, de modo que as medidas terapêuticas deixam de ter os efeitos desejados, resultando em choque irreversível e morte.

\section{METABOLISMO DO OXIGÊNIO}

A hipoxia tecidual, resultante do desequilíbrio entre a oferta e a demanda de oxigênio, acarreta acidose metabólica devido à instalação de metabolismo anaeróbio, que consome mais glicose para produzir menos energia. Enquanto o metabolismo aeróbico produz trinta e oito (38) moles de ATP por mol de glicose consumida, apenas dois (2) moles de ATP são produzidos por mol de glicose, durante a glicólise anaeróbica. $\mathrm{O}$ resultado é a elevação dos níveis séricos de ácido láctico. $\mathrm{O}$ ácido láctico é um indicador de graves reduções na oferta de oxigênio e de déficits energéticos teciduais $^{(1)}$ e está relacionado diretamente com a mortalidade, quando aumenta acima de $2 \mathrm{mmol} / \mathrm{L}^{(2)}$, sobretudo no choque hipovolêmico e cardiogênico. No entanto, seu aumento, no choque séptico, tem um significado duvidoso, conforme discutido anteriormente (no Capítulo II).

\section{MEDIADORES}

As manifestações sistêmicas do choque são o resultado da liberação de uma série enorme de mediadores como citoquinas, eicosanóides, betaendorfinas, toxinas bacterianas, leucotrienos, fator de ativação plaquetária, fator de necrose tumoral, sistema complemento, radicais livres de oxigênio, etc, que provocam disfunção de vários órgãos e sistemas.

1. Disfunção cardíaca: substâncias depressoras do miocárdio contribuem para a depressão da função cardíaca nos choques séptico e hemorrágico, sendo necessário o uso de aminas vasoativas.

2. Disfunção respiratória: há aumento de trabalho respiratório, insuficiência respiratória, edema pulmonar cardiogênico ou não cardiogênico, surgindo a necessidade de suporte ventilatório, mecânico.

3. Disfunção renal: comprometida pelo choque circulatório, pode levar à necrose tubular, aguda. $\mathrm{O}$ uso de drogas nefrotóxicas, contrastes radiológicos e a rabdomiólise aumentam a probabilidade de insuficiência renal, aguda, no choque.
4. Disfunção hepática: a disfunção metabólica inclui prejuízos na atividade depuradora e de síntese, embora sendo transitória e desaparecendo rapidamente com a restauração da perfusão. Manifesta-se por elevação dos níveis de bilirrubinas, transaminases e fosfatase alcalina.

5. Disfunção intestinal: o fluxo sangüíneo esplâncnico é o primeiro a ser desviado para órgãos mais importantes no choque, alterando a permeabilidade intestinal, levando à translocação de bactérias e suas toxinas, contribuindo para a falência orgânica. Manifesta-se por íleo adinâmico, hemorragia do trato gastrintestinal ou diarréia. Outras complicações são a colecistite aguda, alitiásica e, mais raramente, a pancreatite aguda.

6. Disfunção hematológica: freqüentemente observam-se anormalidades na coagulação, podendo culminar com coagulação intravascular, disseminada (trombocitopenia, anemia hemolítica, microangiopática, diminuição do fibrinogênio e aumento de monômeros de fibrina, além de produtos degradados).

\section{DIAGNÓSTICO DO CHOQUE CIRCULATÓRIO}

Três componentes da fisiologia cardíaca são importantes para o reconhecimento do choque:

1. Pré-carga: é representada pela capacitância venosa, que contém $80 \%$ do volume intravascular e pela volemia circulante, efetiva, na prática clínica, é estimada pela pressão venosa, central, média (pré-carga do ventrículo direito) e pela pressão capilar, pulmonar (pré-carga do ventrículo esquerdo) através do uso de cateter de Swan-Ganz;

2. Bomba: a contratilidade cardíaca é que mantém o sistema em atividade, na prática, é medida pela fração de ejeção ventricular ou débito cardíaco;

3. Pós-carga: é a resistência vascular sistêmica (periférica).

O diagnóstico precoce do choque e a instituição de medidas terapêuticas interferem diretamente na morbidade e mortalidade dos pacientes. Sendo assim, após a avaliação e tratamento das vias aéreas e da respiração, o passo seguinte é a avaliação cuidadosa das condições circulatórias. Confiar exclusivamente na pressão sistólica, na avaliação, é muito arriscado, pois os mecanismos compensatórios a mantém em níveis normais até uma perda de $30 \%$ da volemia ${ }^{(3)}$. A atenção deve ser mais dirigida em relação às frequiências cardíaca e respiratória, perfusão cutânea e pressão de pulso (diferença entre a pressão sistólica e diastólica). O rebaixamento do nível de consciência e 
da diurese também são sinais precoces de choque. A frequiência cardíaca varia com a idade e pode estar mantida em níveis normais pelo bloqueio da resposta cardíaca através de drogas como o propranolol e marca-passos. Atletas profissionais, em geral, mantêm freqüência cardíaca baixa, mesmo com perdas volêmicas significativas. A frequiência respiratória está aumentada de modo a combater a acidose metabólica, resultante da hipoxia tecidual, através de alcalose respiratória. A pele é fria e pálida pela vasoconstrição cutânea. A pressão de pulso está diminuída justamente por ação das catecolaminas que aumentam a resistência periférica para compensar a perda volêmica.

\section{CLASSIFICAÇÃO DO CHOQUE}

\subsection{Hipovolêmico}

Pode ser causado por hemorragias, seqüestros, diarréias e perdas cutâneas. É o tipo mais comum nos pacientes traumatizados. Há diminuição do débito cardíaco em virtude da diminuição da pré-carga. O baixo fluxo sangüíneo leva à hipoxia. Como mecanismos de compensação, temos os aumentos da contratilidade, da resistência vascular sistêmica (RVS) e da freqüência cardíaca.

\subsection{Obstrutivo}

Pode ser causado por tromboembolismo pulmonar (TEP), tamponamento cardíaco, pneumotórax hipertensivo e coarctação da aorta. No TEP, ocorre aumento da resistência ao esvaziamento do ventrículo direito, enquanto que, no tamponamento cardíaco, ocorre uma diminuição do enchimento ventricular. No pneumotórax hipertensivo, existe uma diminuição do retorno venoso, levando à diminuição do débito cardíaco. O choque obstrutivo tem como mecanismos compensatórios um aumento da pré-carga, da RVS e da freqüência cardíaca.

\subsection{Distributivo}

Pode ser decorrente de causas neurológicas, como trauma raquimedular, uso de drogas vasodilatadoras, anafilaxia e doenças endócrinas. A vasodilatação ocasiona diminuição da RVS, com diminuição do retorno venoso e do débito cardíaco. Para compensar este estado hemodinâmico, ocorre aumento da contratilidade e da freqüência cardíaca. Nos pacientes em choque, com trauma cranioencefálico, devemos pensar, a princípio, numa causa hipovolêmica.

\subsection{Séptico}

É decorrente de causas infecciosas. Há diminuição da pré-carga pelo seqüestro de líquidos, diminuição da pós-carga por vasodilatação e lesão celular direta. Para compensar, ocorre aumento da freqüência cardíaca e do débito cardíaco.

\subsection{Cardiogênico}

Ocorre devido à falência do ventrículo esquerdo por infarto agudo do miocárdio, cardiopatias e arritmias graves.

O choque hemorrágico (hipovolêmico) é a principal causa de choque em pacientes traumatizados. $\mathrm{O}$ sangramento pode ser externo, óbvio, no caso de lesões arteriais, venosas e fraturas expostas, ou oculto, como nas fraturas de bacia, hematomas retroperitoneais, intraperitoneais ou intratorácicos. O quadro clínico varia de acordo com a gravidade da perda sangüínea, sendo que esta é dividida em classes para facilitar sua compreensão, embora nem sempre isto seja possível. Na Tabela I, citamos como exemplo um homem adulto de $70 \mathrm{~kg}$, com volemia aproximada de cinco (5) litros.

O tratamento do choque hemorrágico é melhor orientado pela resposta individual do que pela classificação descrita. Assim, os mesmos sinais e sintomas utilizados para o diagnóstico são úteis para avaliar sua resposta. A normalização do pulso, pressão de pulso, pressão arterial, débito urinário, freqüência respiratória, da consciência e do equilíbrio ácido-base indicam resposta adequada. A dosagem do ácido láctico é útil na avaliação da perfusão tecidual.

As causas de choque não hemorrágico podem ser devidas a: falência miocárdica (cardiogênico), pneumotórax hipertensivo, choque neurogênico e choque séptico:

1. Falência Miocárdica (Cardiogênico): pode ocorrer por contusão do miocárdio, tamponamento cardíaco, embolia gasosa, arritmias ou até infarto agudo do miocárdio. O tamponamento cardíaco (TC) é mais comum nas lesões penetrantes de tórax e ocorre quando um determinado volume de sangue, contido no saco pericárdico, inibe a atividade cardíaca. O quadro clínico do TC é de taquicardia, abafamento de bulhas, ingurgitamento das veias do pescoço, hipotensão, aumento da PVC. O tratamento consiste na pericardiocentese e toracotomia de emergência para correção da lesão. 


\begin{tabular}{|c|c|c|c|c|}
\hline & Classe I & Classe II & Classe III & Classe IV \\
\hline \multirow{2}{*}{$\begin{array}{l}\text { Perda sangüínea (mL) } \\
\text { perda sangüínea (\% vs) }\end{array}$} & até 750 & $750-1500$ & $1500-2000$ & $>2000$ \\
\hline & até $15 \%$ & $15-30 \%$ & $30-40 \%$ & $>40 \%$ \\
\hline \multirow{3}{*}{$\begin{array}{l}\text { freqüência de pulso } \\
\text { pressão arterial } \\
\text { pressão de pulso }(\mathrm{mmHg})\end{array}$} & $<100$ & $>100$ & $>120$ & $>140$ \\
\hline & normal & normal & diminuída & diminuída \\
\hline & normal/aumentada & diminuída & diminuída & diminuída \\
\hline freqüência respiratória & $14-20$ & $20-30$ & $30-40$ & $>35$ \\
\hline diurese $(\mathrm{mL} / \mathrm{h})$ & $>30$ & $20-30$ & $5-15$ & desprezível \\
\hline estado mental/SNC & ansiedade leve & ansiedade moderada & ansiedade e confusão & confusão e letargia \\
\hline reposição volêmica & cristalóide & cristalóide & cristalóide e sangue & cristalóide e sangue \\
\hline
\end{tabular}

2. Pneumotórax hipertensivo: se desenvolve quando há vazão unidirecional de ar para o espaço pleural, decorrente de lesão, ou da parede torácica, ou do parênquima pulmonar. $\mathrm{O}$ acúmulo de ar é progressivo, levando ao colabamento do pulmão do lado afetado, ao desvio das estruturas do mediastino para o lado oposto, colabamento do pulmão contralateral, compressão das câmaras cardíacas e impedimento do retorno venoso ao coração. O quadro clínico se assemelha ao do TC, quando ocorre do lado esquerdo do tórax, mas, como é mais freqüente, deve ser descartado em primeiro lugar. Ocorre abolição do murmúrio vesicular do lado afetado, desvio da traquéia para o lado oposto, estase jugular, hipotensão, taquicardia, enfisema subcutâneo e insuficiência respiratória aguda. O diagnóstico é clínico, nunca radiológico, pois representa uma condição de risco de vida iminente. O tratamento consiste na imediata descompressão torácica através da inserção de agulha de grosso calibre no segundo espaço intercostal, na linha hemiclavicular, de modo a transformá-lo em pneumotórax simples, seguindo-se a drenagem pleural, convencional, com tubo de grosso calibre, ao nível da linha do mamilo, anterior à linha axilar média.

3. Choque neurogênico: as lesões cranianas isoladas não causam choque. Uma lesão medular pode provocar hipotensão com perda do tônus simpático, acentuando a hipovolemia. O quadro clínico é de hipotensão arterial, sem taquicardia e sem vasoconstrição cutânea. $\mathrm{O}$ tratamento inicial consiste na reposição volêmica, descartando-se causas hemorrágicas para o choque.
4. Choque séptico: é mais comum ocorrer naqueles pacientes que chegam ao hospital após várias horas do trauma. As causas, em geral, são lesões de vísceras ocas, que causam contaminação da cavidade peritoneal. Pode, também, ser decorrente de causas clínicas como pneumonia, meningite, colecistite, pancreatite e pielonefrite. O quadro clínico é muito parecido com o choque hemorrágico, sendo que seu tratamento inicial é direcionado à reposição volêmica.

A resposta do doente à reposição volêmica inicial é a chave para se determinar a terapêutica subseqüente (Tabela II).

\section{TRATAMENTO DO CHOQUE}

A própria definição do choque, como uma redução crítica na perfusão tecidual, por anormalidade do sistema circulatório, já se transforma em instrumento operacional, tanto para o seu diagnóstico quanto para o seu tratamento. Ao se conduzir o tratamento dos pacientes em choque circulatório, as duas prioridades são a rápida avaliação do processo patológico e a obtenção de estabilidade cardiopulmonar. As intervenções sobre esta última baseiam-se na ventilação, infusão e bombeamento.

Para os pacientes traumatizados, o processo de identificação do choque está diretamente relacionado ao mecanismo de lesão. Todos os tipos de choque podem estar presentes no paciente traumatizado. A sistematização do atendimento inicial é fundamental, de modo que o diagnóstico das anormalidades e seu 


\begin{tabular}{|c|c|c|c|}
\hline & Resposta rápida & Resposta transitória & Sem resposta \\
\hline sinais vitais & retorno ao normal & $\begin{array}{l}\text { melhora transitória; } \\
\text { recidiva PA/ FC }\end{array}$ & continuam anormais \\
\hline perda sangüínea estimada & mínima (10-20\%) & $\begin{array}{c}\text { moderada e persistente } \\
(20-40 \%)\end{array}$ & grave $(>40 \%)$ \\
\hline $\begin{array}{l}\text { necessidade de mais } \\
\text { cristalóide }\end{array}$ & baixa & alta & alta \\
\hline necessidade de sangue & baixa & moderada-alta & imediata \\
\hline preparo de sangue & tipo e com prova cruzada & tipo específico & $\begin{array}{c}\text { liberado em caráter de } \\
\text { emergência }\end{array}$ \\
\hline necessidade de cirurgia & possível & provável & muito provável \\
\hline $\begin{array}{l}\text { necessidade de opinião } \\
\text { cirúrgica }\end{array}$ & $\operatorname{sim}$ & $\operatorname{sim}$ & $\operatorname{sim}$ \\
\hline
\end{tabular}

tratamento são feitos passo-a-passo, numa sequiência lógica. Além disso, as prioridades são as mesmas para qualquer tipo de choque.

Na Unidade de Emergência do Hospital das Clínicas da Faculdade de Medicina de Ribeirão Preto, as disciplinas de Cirurgia de Urgência e de Terapia Intensiva do Departamento de Cirurgia, Ortopedia e Traumatologia seguem as normas do Comitê de Trauma do Colégio Americano de Cirurgiões, através do manual do Advanced Trauma Life Support-ATLS ${ }^{(3)}$. Dá-se prioridade ao "ABC" onde a letra "A"(do inglês airway) corresponde ao acesso às vias aéreas, de modo a mantê-las prévias e protegidas contra obstrução por corpos estranhos e queda da língua, aspiração brônquica de conteúdo gástrico, sangue, fragmentos de dentes, etc. A letra "B" corresponde à respiração (do inglês breathing). A ventilação e oxigenação são mantidas através de altos fluxos de oxigênio, fornecendo altas concentrações de $\mathrm{O}_{2}$ no ar inspirado. A ventilação mecânica é instituída quando a ventilação por máscara não é suficiente. O suporte ventilatório mecânico reduz as necessidades metabólicas por reduzir o trabalho respiratório do paciente, permitindo a redistribuição do fluxo sangüíneo para áreas hipoperfundidas. Os sinais mais claros de insuficiência respiratória são a taquipnéia e a respiração superficial, com batimento de asas do nariz. Além disso, as medidas dos gases arteriais podem orientar a conduta (de modo geral, indica-se intubação endotraqueal, quando a $\mathrm{PaO}_{2}<50 \mathrm{mmHg}$ mesmo com máscara de
$\mathrm{O}_{2}$ ou $\mathrm{PaCO}_{2}>50 \mathrm{mmHg}$ ). O pneumotórax hipertensivo compromete tanto a ventilação quanto o sistema circulatório. Entretanto, as dificuldades de ventilação e oxigenação pelas alterações da mecânica respiratória coloca a vida do paciente em risco, de modo mais rápido do que a falta de um volume circulante efetivo. Assim, o tratamento consiste, inicialmente, na drenagem pleural imediata, seguida ou não de reposição volêmica, agressiva. Em seguida, dá-se prioridade à letra "C' $\mathbf{C}$ " que corresponde à manutenção da circulação. A hipovolemia grave está presente na grande maioria com causas clínicas e cirúrgicas de choque circulatório e em até um terço dos pacientes com infarto agudo do miocárdio e hipoperfusão ${ }^{(4)}$. Conseqüentemente, a rápida infusão de líquidos é necessária para expandir o volume intravascular e restaurar o retorno venoso. A terapia com líquidos está em relação direta com os parâmetros específicos do desempenho circulatório restaurado, como a freqüência cardíaca, pressão arterial, pressão venosa, central (PVC), débito cardíaco (DC), diurese e depuração do lactato arterial. A medida da PVC permite estimar a pressão de enchimento do ventrículo direito (VD), constituindo-se numa maneira prática, rápida e barata de se estimar o grau da volemia do paciente e a necessidade ou não do uso de drogas vasoativas. Embora em algumas situações as pressões do VD não sejam o reflexo direto das pressões do ventrículo esquerdo (insuficiências ou estenoses valvulares importantes, embolia pulmonar e aumentos da resistência 
vascular, pulmonar), o uso da PVC está formalmente indicado nos casos de choque ou hipotensão grave, permitindo a detecção de hipo ou hipervolemia, assim como indícios de falência miocárdica. A cateterização da artéria pulmonar, método mais refinado, através do cateter de Swan-Ganz, fornece informações precisas acerca do débito cardíaco, pressão de enchimento do VE e pressão capilar, pulmonar (PCP). A PCP consiste em variável importante na quantificação da pressão hidrostática e da pré-carga do VE. Entretanto, é uma técnica bastante onerosa, exigindo local apropriado, equipe bem treinada e equipamento de alto custo. As medidas da PVC, através da prova de expansão volêmica, aliadas ao quadro clínico, são úteis na avaliação rápida do estado cardiocirculatório de um paciente grave ${ }^{(5)}$. A prova de expansão volêmica consiste, inicialmente, na introdução de um cateter central, posicionado, em geral, próximo ao átrio direito através da veia jugular interna ou da subclávia. $\mathrm{O}$ valor normal da PVC situa-se entre 8 e $12 \mathrm{~cm}$ de água, quando o ponto zero é fixado na linha axilar média, com o paciente calmo, em decúbito dorsal, horizontal e em ventilação espontânea e tranquiila. A prova de expansão volêmica tem por finalidade analisar o papel do miocárdio frente à administração rápida de uma quantidade de volume (em geral $10 \%$ da volemia). Infunde-se, pelo cateter venoso, central, cerca de 300 a $500 \mathrm{~mL}$ de ringer simples em vinte (20) minutos. Mede-se imediatamente a PVC. As possíveis variações são as relacionadas a seguir.

Situação 1: é a mais freqüente. Geralmente, ocorre no pós-operatório imediato, na desidratação leve e em hemorragias de classe I. A PVC inicial é normal ou discretamente baixa e, após o teste, a PVC e a diurese se normalizam.

Situação 2: as perdas líquidas são maiores, de modo que há necessidade de infusão de quantidades adicionais de líquidos após o teste, a fim de restabelecer o estado hemodinâmico. Caso haja demora no retorno da diurese, pode-se, a partir daí, utilizar diuréticos de ação rápida, como o furosemide. Caso a demora se prolongue, pode ser útil a utilização de dopamina em doses diuréticas.

Situação 3: é característica das hemorragias traumáticas de classe III e IV, onde o tempo para restabelecimento dos parâmetros hemodinâmicos é maior e com necessidade de utilização de hemoderivados. A partir da normalização da PVC é que se pode pensar na utilização de diuréticos e aminas vasoativas.
Situação 4: ocorre ascensão rápida da PVC ( 5 a $7 \mathrm{~cm}$ acima do valor basal) devido ao comprometimento miocárdico, com necessidade precoce de diuréticos e aminas vasoativas (dopamina, dobutamina), a fim de melhorar as condições hemodinâmicas.

Situação 5: é a situação mais grave, onde o componente cardiogênico exerce papel fundamental, e, se não tratado de forma adequada, evolui para choque irreversível. A PVC inicial já se encontra em níveis elevados (acima de $12 \mathrm{~cm}$ de água). A reposição volêmica deve ser feita com muita cautela e o uso de aminas simpatomiméticas, mais notadamente a dobutamina, é mandatória. Nesses casos, há indicação de monitorização hemodinâmica, invasiva. A Figura 1 ilustra as situações descritas acima.

No atendimento inicial ao paciente traumatizado, inicia-se a infusão rápida, no adulto, de um (1) a dois (2) litros e, na criança, de $20 \mathrm{~mL} / \mathrm{kg}$ de peso corporal de cristalóide (soro fisiológico a $0,9 \%$ ou ringer lactato). A resposta hemodinâmica norteia o passo seguinte. Se não houver melhora, repete-se o passo anterior. Persistindo a instabilidade hemodinâmica, já está indicada a reposição com concentrado de hemácias, de preferência tipo específico, ou em condições de emergência, tipo $\mathrm{O}$ negativo. O controle do choque hemorrágico é feito através da restauração rápida da volemia concomitantemente ao combate do foco de sangramento. Para os casos com resposta hemodinâmica transitória ou sem resposta à reposição, a necessidade de cirurgia torna-se mais provável para o controle do foco hemorrágico.

Nos pacientes com choque cardiogênico ou séptico, ou naqueles com doença cardíaca subjacente, a monitorização hemodinâmica, invasiva, com cateterização da artéria pulmonar deve ser empregada. A escolha do líquido a ser infundido ainda é controvertida. Tanto as soluções cristalóides quanto as colóides devem ser utilizadas para restaurar a volemia, além de sangue e seus derivados, quando necessários. De modo geral, é necessária a administração de grandes quantidades de líquidos, via intravenosa, para os casos de choque, sendo bem tolerada quando a permeabilidade vascular está mantida. Entretanto, em situações de aumento da permeabilidade capilar, como no trauma, sepse e reação anafilática, a infusão de líquidos acarreta um edema intersticial que pode afetar algumas funções orgânicas, como: edema cerebral com alterações neurológicas, edema pulmonar com alteração das trocas gasosas, edema intestinal com 


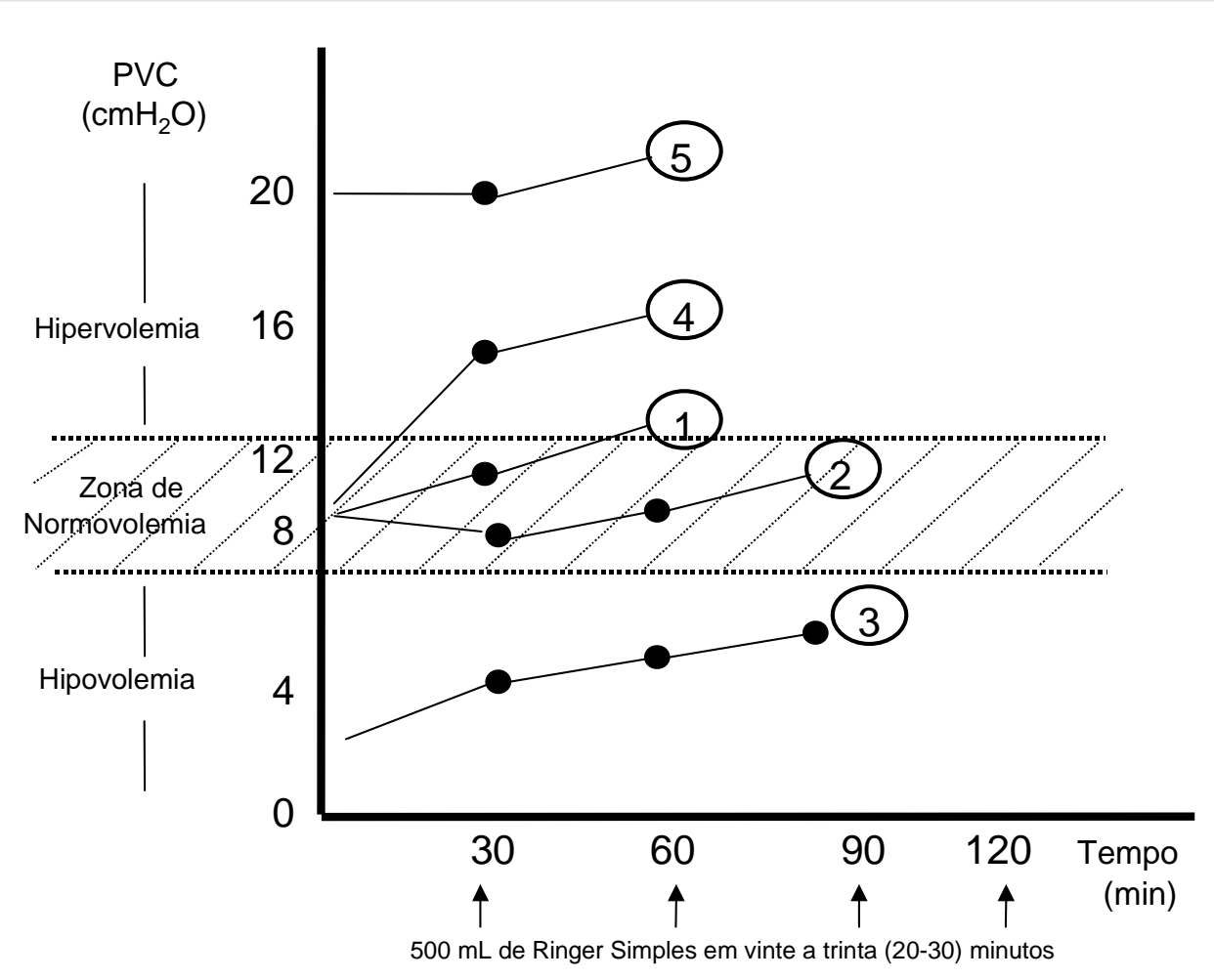

Figura 1 - A prova de expansão volêmica.

alteração na absorção de nutrientes e translocação bacteriana, edema de partes moles, favorecendo lesões superficiais, com dificuldade de cicatrização. Os colóides podem reduzir o risco de edema pulmonar, pela manutenção da pressão coloidosmótica, plasmática, durante a reposição volêmica. Os preparados com soluções colóides e solução salina hipertônica também estão sendo empregados. A hipernatremia, hipertonicidade e maior custo são os principais riscos e desvantagens do seu uso. Os cristalóides também são eficazes, mais baratos, mas levam à hemodiluição, comprometendo substancialmente a oferta global de oxigênio sistêmico, se o débito cardíaco não estiver significativamente aumentado pela melhora da pré-carga. Em geral, procura-se manter o hematócrito em $30 \%$ e a taxa de hemoglobina em $10 \mathrm{~g} / \mathrm{dL}$. Os mais jovens, geralmente, têm condições de suportar taxas menores (até 5 a $7 \mathrm{~g} / \mathrm{dL}$.), seguindo a tendência mundial de evitar expô-los aos riscos das transfusões sangüíneas.

A seguir, os tipos de soluções disponíveis para reposição volêmica.

\subsection{Cristalóides}

São soluções pouco onerosas e facilmente acessíveis. As mais utilizadas são o ringer lactato e o soro fisiológico a $0,9 \%$. Estas soluções se equilibram livremente entre os espaços intravascular e intersticial, sendo eficazes em expandir ambos os compartimentos. Seu efeito hemodinâmico é máximo ao final da infusão, mas tem curta duração. Após a infusão destas soluções, a expansão plasmática é da ordem de $25 \%{ }^{(6)}$. Evidências atuais indicam que a ressuscitação com cristalóides está associada a reduções na pressão coloidosmótica e, em alguns casos, ao edema pulmonar, sistêmico e cerebral. A ressuscitação com colóides, que mantêm a pressão coloidosmótica, parece não predispor à formação de edema. Mitchell et al. em $1992^{(7)}$, descreveram, em pacientes críticos, uma correlação negativa entre a sobrevida e o balanço hídrico positivo. A manutenção da estabilidade hemodinâmica requer duas (2) a seis (6) vezes o volume de cristalóides, quando comparados ao de colóides ${ }^{(8)}$. Os cristalóides são as soluções mais utilizadas para a reanimação inicial do choque hemorrágico, de acordo com as normas do ATLS ${ }^{(3)}$. 


\subsection{Soluções salinas hipertônicas}

Recentemente, tem-se discutido muito o uso de solução salina, hipertônica, a 7,5\%, isolada ou associada ao colóide dextran 70. Estas soluções exercem pressão osmótica importante na membrana celular, com redistribuição do fluido intracelular para o compartimento extracelular. As soluções salinas hipertônicas têm sido indicadas na ressuscitação pré-hospitalar do choque hemorrágico devido a sua capacidade superior em expandir o volume sangüíneo, elevar a pressão arterial e o débito cardíaco com volumes pequenos, cujo efeito é praticamente instantâneo, com duração de até quatro horas. Entretanto, alguns estudos têm demonstrado piora dos parâmetros hemodinâmicos e maior mortalidade, quando este tipo de solução é utilizada em hemorragias sem controle do foco hemorrágico ${ }^{(9)}$. Parece haver efeito benéfico sobre a pressão intracraniana nos pacientes com choque e trauma cranioencefálico, quando comparado ao uso de ringer lactato $^{(10)}$. A sua principal desvantagem é o risco de hipernatremia e hiperosmolaridade. Apesar das suas aparentes vantagens, as soluções salinas, hipertônicas não devem ser utilizadas como opção de rotina no tratamento do choque.

\subsection{Colóides}

Existem muitas suspensões coloidais disponíveis, com variedade no tamanho das moléculas, meia-vida, pressão coloidosmótica, efeitos colaterais e custo. Os agentes coloidais mais comumente utilizados para reposição volêmica incluem a albumina humana, o dextran, o hidroxietilamido (HES) e o pentaamido.

\subsubsection{O plasma fresco congelado (PFC)}

Não é recomendado para reposição volêmica, segundo normas de consenso norte-americanas ${ }^{(11)}$. O PFC foi descrito como o hemoderivado mais utilizado de maneira incorreta (aproximadamente $90 \%$ dos casos). Indicações inaceitáveis são o seu uso como expansor de volume, fonte de imunoglobulina, exceto em raras circunstâncias, fonte de nutrição ou de fibronectina. Indicações aceitáveis incluem o tratamento de defeitos de coagulação pós-reposição e tratamento da intoxicação por warfarin, com risco de vida. Além disso, o PFC é caro, tem poder oncótico igual ou menor que as soluções descritas anteriormente e tem o risco de transmissão de doenças como citomegalovírus, SIDA e hepatite.

\subsubsection{Albumina}

É responsável por 60 a $80 \%$ da pressão oncótica, plasmática, normal. É cara por ser derivada de um "pool" de plasma humano. Seus níveis estão mais relacionados a fatores prognósticos do que à necessidade de mantê-los em níveis adequados através da infusão de albumina exógena. Pode ser utilizada a 5 e $25 \%$ e está indicada, principalmente, quando há risco de distúrbios da coagulação, onde o uso dos outros colóides pode desencadear coagulopatias ${ }^{(12)}$.

\subsubsection{Dextran}

É um agente coloidal, polidisperso, preparado a partir de polímeros de glicose. Comercialmente, existem dois produtos: o dextran 40 e o 70 . O dextran 40 é preparado sob a forma de solução hiperoncótica a $10 \%$, com peso médio de suas moléculas de 40.000 dáltons, tendo meia-vida curta, porque muitas de suas moléculas são pequenas. O efeito hiperoncótico produz uma expansão inicial do volume intravascular, que é maior que o volume administrado. $\mathrm{O}$ dextran 70 é um colóide polidisperso, com média ponderada de pesos moleculares de 70.000 dáltons. Tem um tempo de retenção vascular maior que o dextran 40. Ambos os dextrans podem melhorar a circulação microvascular por diminuir a viscosidade sangüínea e minimizar a agregação de plaquetas e hemácias. Entretanto, podem causar sangramentos pelo mesmo mecanismo e estão associados a 0,03 a $0,07 \%$ de anafilaxia $^{(13)}$. Para evitar estes efeitos adversos, recomenda-se não ultrapassar o volume de $1,5 \mathrm{~g} / \mathrm{kg} / \mathrm{dia}$ (1500 mL/dia).

\subsubsection{Hetamido (HES - hidroxyetyl starch ou hidroxietilamido)}

É um amido natural de polímeros de glicose, derivados da amilopectina. É composto por moléculas com média ponderada de 45.000 dáltons, tendo uma meia-vida plasmática de aproximadamente dezessete (17) dias e pressão oncótica de $28 \mathrm{mmHg}$. Está disponível em solução a $6 \%$ e $10 \%$, sendo que sua degradação produz produtos que são excretados pela urina e fezes. Os efeitos adversos do HES são similares aos de outros colóides e incluem possíveis reações anafilactóides, precipitação de insuficiência cardíaca, congestiva ou insuficiência renal e alterações da coagulação. É difícil recomendar a dosagem máxima segura, pois a resposta de cada paciente é variável e as doses recomendadas pelo fabricante de $33 \mathrm{~mL} / \mathrm{kg} /$ dia para o HES $10 \%$ e $20 \mathrm{~mL} / \mathrm{kg} /$ dia para 
HES 6\% não são suportadas por dados publicados ${ }^{(14)}$ Entretanto, é uma alternativa ao uso da albumina como expansor volêmico, em várias situações clínicas e com menor custo.

\subsubsection{Pentaamido}

É uma formulação única mais recente do HES, com média ponderada de pesos moleculares de 280.000 dáltons, com meia-vida plasmática de dez (10) horas e pressão oncótica de $40 \mathrm{mmHg}$. O pentaamido produz um grau maior de expansão do volume intravascular do que o HES ou albumina devido à sua maior pressão oncótica. Apresenta, também, possíveis efeitos adversos semelhantes ao HES, porém, em geral, menos extensos.

\subsubsection{Gelatina}

Devido à sua diferença insignificante de capacidade de expansão volêmica, quando comparada aos cristalóides e aos potenciais riscos de reações anafiláticas, não são mais utilizadas em países desenvolvidos (Tabela III).

A monitorização eletrocardiográfica pode mostrar arritmias que devem ser tratadas rapidamente através de tratamento farmacológico e, dependendo do aparecimento de instabilidade hemodinâmica, através de cardioversão. As bradiarritmias, que não respondem ao uso de atropina ou isoproterenol, necessitam de estimulação endocavitária temporária.
A função cardíaca inadequada e a hipoperfusão persistente, após reposição da volemia, podem ser tratadas com o uso de aminas vasoativas. Entretanto o uso excessivo pode afetar de forma adversa o metabolismo do oxigênio sistêmico, do miocárdio e a perfusão tecidual. Não há regras nem doses uniformes para o seu uso. No entanto, é consenso que seu uso só se torna benéfico após a restauração da volemia, sendo que a dose deve ser ajustada para cada caso, começando-se a infundir sempre a menor dose possível, de modo a diminuir seus efeitos indesejáveis. As principais drogas utilizadas são a dobutamina, dopamina e noradrenalina, além das drogas vasodilatadoras. A dopamina entre 2 e $5 \mu \mathrm{g} / \mathrm{kg} / \mathrm{min}$ é empregada isoladamente ou em combinação com outros inotrópicos para aumentar a perfusão renal e estimular a diurese, podendo atenuar efeitos deletérios de vasoconstritores como a noradrenalina. Provoca, também, vasodilatação mesentérica, coronariana e cerebral. Entre $6 \mathrm{e}$ $25 \mu \mathrm{g} / \mathrm{kg} / \mathrm{min}$, sua ação é predominantemente beta, causando aumento do débito cardíaco por aumento da frequiência cardíaca, do retorno venoso e diminuição da resistência vascular periférica. Em doses ainda maiores, provoca vasoconstrição periférica ainda maior e queda da diurese, com aumento da pressão arterial. Está indicada em situações de oligúria e choque. Como efeitos adversos, têm-se aumento do "shunt" pulmonar com piora da hipoxia, náuseas, vômitos, taquicardias, arritmias ventriculares e angina. A dobutamina está indicada nos casos de choque e

\begin{tabular}{|c|c|c|c|c|c|c|c|c|c|}
\hline \multicolumn{10}{|c|}{ Tabela III - Características dos líquidos descritos } \\
\hline & SF $0,9 \%$ & $\begin{array}{l}\text { ringer } \\
\text { lactato }\end{array}$ & $\begin{array}{c}\text { solução salina } \\
\text { hipertônica } \\
7,5 \%\end{array}$ & $\begin{array}{c}\text { albumina } \\
25 \%\end{array}$ & $\begin{array}{l}\text { albumina } \\
5 \%\end{array}$ & $\begin{array}{l}\text { hetaami- } \\
\text { do } 6 \%\end{array}$ & $\begin{array}{l}\text { dextran } 40 \\
(10 \%)\end{array}$ & $\begin{array}{c}\text { dextran } 70 \\
(6 \%)\end{array}$ & PFC \\
\hline sódio (mEq/L) & 154 & 130 & 1250 & $130-160$ & $130-160$ & 154 & 154 & 154 & 170 \\
\hline cloro (mEq/L) & 154 & 109 & 1250 & $130-160$ & $130-160$ & 154 & 154 & 154 & 100 \\
\hline Osmolaridade (mOsm/L) & 310 & 275 & 2400 & 1500 & 310 & 310 & 310 & 310 & 300 \\
\hline pressão oncótica (mmHg) & 0 & 0 & 0 & 100 & 20 & 30 & - & 60 & 20 \\
\hline lactato (mEq/L) & 0 & 28 & 0 & 0 & 0 & 0 & - & 0 & 4 \\
\hline $\begin{array}{l}\text { expansão plasmática } \\
\text { após } 500 \mathrm{~mL}\end{array}$ & 90 a 100 & $80-100$ & $700 / 100$ & $1500-200$ & 500 & $500-700$ & $500-1000$ & $500-700$ & - \\
\hline duração do efeito (horas) & - & - & 4 & $<24$ & $<24$ & $<36$ & $<6$ & $<24$ & - \\
\hline $\mathrm{PH}$ & 5,0 & 6,5 & 5,5 & 6,9 & 6,9 & 5,5 & - & $3,0-7,0$ & variável \\
\hline
\end{tabular}


insuficiência cardíaca, nas doses entre 5 a $15 \mu \mathrm{g} / \mathrm{kg} / \mathrm{min}$, causando aumento do débito cardíaco e vasodilatação periférica. Seus efeitos adversos incluem arritmias cardíacas, cefaléia, ansiedade, tremores, hiper ou hipotensão arterial. A noradrenalina é utilizada em doses entre 0,02 a $0,15 \mu \mathrm{g} / \mathrm{kg} / \mathrm{min}$, provocando vasoconstrição periférica, sendo indicada no choque séptico. Os efeitos adversos são decorrentes da vasoconstrição: queda da diurese, necrose e ulcerações cutâneas e hemorragias cerebrais.

Não há consenso sobre o uso de bicarbonato de sódio no tratamento do choque com acidose láctica. Estudos recentes ${ }^{(15,16)}$ demonstram não ocorrer melhora dos parâmetros hemodinâmicos, em pacientes críticos que apresentam acidose metabólica e elevação do ácido láctico.
Finalmente, o uso de agentes antimicrobianos e intervenções cirúrgicas adequadas devem ser utilizados para os pacientes com choque séptico. A embolia pulmonar maciça deve ser tratada com agentes trombolíticos ou cirurgia. A monitorização hemodinâmica invasiva também é útil na avaliação de parâmetros de oxigenação, otimizando a oferta e a demanda de $\mathrm{O}_{2}$. No doente crítico, a oxigenação sistêmica, adequada é fundamental para a recuperação desses parâmetros. Os cálculos de oxigenação e a calorimetria indireta ajudam a caracterizar a eficiência da oxigenação do sangue e as taxas de transporte e consumo de $\mathrm{O}_{2}$. As variáveis mais freqüentemente utilizadas são: a oferta de $\mathrm{O}_{2}\left(\mathrm{DO}_{2}\right), \mathrm{o}$ consumo de $\mathrm{O}_{2}\left(\mathrm{VO}_{2}\right)$ e a taxa de extração de $\mathrm{O}_{2}$ $\left(\mathrm{O}_{2}\right.$ ER).

MARSON F et al. Circulatory shock syndrome. Medicina, Ribeirão Preto, 31: 369-379, july/sept. 1998.

ABSTRACT: Circulatory shock is marked by critical reductions on tecidual perfusion, causing severe systemic alterations, impairing cellular and organic function, with a high mortality rate as result. The prompt diagnosis and therapeutics should be based on individual response, emphasizing ventilatory and hemodynamic support. The knowledge of physiopathology directs therapeutic decisions, since the objectives to be reached are based on physiopathology. Several recent therapies are being investigated on patients with circulatoty shock, such as fluid resuscitation, red cells bood substitutes, and therapies directed to mediators (cytokines, endotoxins, prostaglandins, leukotrienes, and platelet-activating factor).

UNITERMS: Shock. Hemodynamics.

\section{REFERÊNCIAS BIBLIOGRÁFICAS}

1 - HAUPT $M$ et al. Fluid loading increases oxygen consumption in septic patients with lactic acidosis. Am Rev Respir Dis 131: 912-916, 1985.

2 - CADY L et al. Quantification of critical illness with special reference to blood lactate. Crit Care Med 1: 75-80, 1973.

3 - ADVANCED TRAUMA LIFE SUPPORT. Doctors instructor manual. 6 th ed. American College of Surgeons, Chicago, 1997, 99op.

4 - FORRESTER JS et al. Correlative classification of clinical and hemodynamic function after acute myocardial infarction. Am J Cardiol 31: 137-145, 1977.

5 - BASILE FILHO A et al. A prova de expansão volêmica, através da pressão venosa central, pode ser utilizada para racionalizar os custos e o uso de drogas vasoativas nos pacientes graves? Arq Bras Med 69: 77-79, 1995.

6 - FALK JL et al. Fluid resuscitation in shock. J Cardiothorac Anesth 2: 33-38, 1988.
7 - MITCHELL JP et al. Improved outcome based on fluid management in critically ill patients requiring pulmonary artery catheterization. Am Rev Resp Dis 145: 990-998, 1992.

8 - TRAYLOR RJ \& PEARL RG. Crystalloid versus colloid versus colloid: all colloids are not created equal. Anesth Analg 83: 209-212, 1996.

9 - KRAUSZ MM. Controversies in shock research: hypertonic resuscitation- pros and cons. Shock 3: 69-72, 1995.

10 - ANDERSON JT et al. Initial small-volume hypertonic resuscitation of shock and brain injury: short- and long-term effects. J Trauma 42: 592-600, 1997.

11 - GOULD SA et al. Choque hipovolêmico. In: RACCKOW EC \& ASTIZ ME. Choque circulatório, Interlivros, Rio de Janeiro, p. 237-257, 1993.

12 - BOLDT $\mathrm{J}$ et al. Influence of different volume terapies on platelet function in the critically ill. Intensive Care Med 22: 1075-1081, 1996.

13 - IMM A \& CARLSON RW. Reposição volêmica no choque circulatório. In: RACKOW EC \& ASTIZ ME. Choque circulatório. Interlivros, Rio de Janeiro, v. 2, p. 311-331, 1993. 
14 - WARREN BB \& DURIEUX ME. Hydroxyethyl Starch: safe or not? Anesth Analg 84: 206-212, 1997.

15 - COOPER DJ et al. Bicarbonate does not improve hemodynamics in critically ill patients who have lactic acidosis. A prospective, controlled clinical study. Ann Intern Med 112: 492-498, 1990.
16 - MATHIEU D et al. Effects of bicarbonate on hemodynamics and tissue oxygenation in patients with lactic acidosis: a prospective, controlled clinical study. Crit Care Med 19: 1352-1356,1991.

Recebido para publicação em 04/03/98

Aprovado para publicação em 13/05/98 